\title{
Proof That Spatial Transitions Release/Absorb Energy That Compactification Necessarily Leads to Changes in Volume, Energy and Entropy
}

\author{
Christopher Pilot \\ Physics Department Gonzaga University, Spokane, WA, USA \\ Email: pilot@gonzaga.edu
}

How to cite this paper: Pilot, C. (2019) Proof That Spatial Transitions Release/Absorb Energy That Compactification Necessarily Leads to Changes in Volume, Energy and Entropy. Journal of High Energy Physics, Gravitation and Cosmology, 5, 310-320. https://doi.org/10.4236/jhepgc.2019.52017

Received: January 8, 2019

Accepted: February 12, 2019

Published: February 15, 2019

Copyright () 2019 by author(s) and Scientific Research Publishing Inc. This work is licensed under the Creative Commons Attribution International License (CC BY 4.0).

http://creativecommons.org/licenses/by/4.0/

\begin{abstract}
Using simple box quantization, we demonstrate explicitly that a spatial transition will release or absorb energy, and that compactification releases latent heat with an attendant change in volume and entropy. Increasing spatial dimension for a given number of particles costs energy while decreasing dimensions supplies energy, which can be quantified, using a generalized version of the Clausius-Clapyeron relation. We show this explicitly for massive particles trapped in a box. Compactification from $N$-dimensional space to $(N-1)$ spatial dimensions is also simply demonstrated and the correct limit to achieve a lower energy result is to take the limit, $L_{w} \rightarrow 0$, where $L_{w}$ is the compactification length parameter. Higher dimensional space has more energy and more entropy, all other things being equal, for a given cutoff in energy.
\end{abstract}

\section{Keywords}

Spatial Transitions, Box Quantization, Compactification

\section{Introduction}

Compactification is an old idea [1] [2] where one reduces the dimension of space to account for observed symmetries and conservation laws. In the very earliest versions, this was seen as a mechanism to unify electromagnetism with gravity. The concept has since been extended [3] [4] [5] [6] in the modern era to include supergravity and superstring theory, which seeks to accommodate the known particle spectrum of elementary particles and known conservation laws 
under one grand unification scheme. These Kaluza-Klein theories entertain dimensions as high as 10 and even 26-dimensional space, and often predict particles which are yet to be observed, such as supersymmetric partners. The underlying symmetries are also new, and reduce in a low energy limit, to the known weak-electromotive forces, gravity and QCD.

Temperature must play a key role in such theories. Compactification is thought to occur at very high temperatures, i.e. very short distance scales. It seems to us that a thermodynamic treatment is therefore necessary. Pressure is also of fundamental importance, as we are dealing with massive particles and mediating fields in the very early universe. Recently [7], we generalized the Clausius-Clapyeron (CC) relation to allow for a phase transition involving a change in spatial dimension at a given temperature and pressure. Using black-body radiative photons as our substance, this generalization allowed us to quantify the latent heat given off in transitioning from $N$-spatial dimensions to $(N-1)$ dimensions, and the latent heat absorbed in going from $(N-1)$ spatial dimensions to $N$ dimensions. Moreover, expressions for the changes in entropy and volume were derived when undergoing this type of first order discontinuous phase transition.

To illustrate the generalized CC relation, we also gave a specific numerical example, considering a temperature applied to the very early universe. It was conjectured that, based on the explosive release of heat energy, and the attendant changes in entropy and volume, the $N=4$ to $(N-1)=3$ transition may have a connection to inflation. The universe may have underwent a phase transition from 4-space to 3-space within the earliest times [8]. We argued [7] that this would bypass the need for the inflaton field, as well as doing away with a-causal expansion. If it is the spatial dimension itself, which is changing, i.e. undergoing a phase transition, a-causality within a given fixed space becomes a secondary issue. Finally, quantum mechanical fluctuations about a mean temperature were also discussed when transitioning between spatial dimensions. It was shown that if the phase transition involves the same thermodynamic process on both sides of the co-existence curve, such as from adiabatic expansion to adiabatic expansion, then the temperature fluctuations, $\delta T / T$, carry through unperturbed from one space to the neighboring space. If, on the other hand, the phase transition involves a difference in thermodynamic process on either side of the coexistence curve, such as from isothermal expansion to adiabatic expansion, then it was discovered that the thermal fluctuations can be created within the transition itself.

In this short note, we pursue this analysis further and prove that space is equivalent to energy another way. We focus on box quantization and specialize to massive particles, such as electrons. We consider what happens in the simplest case of a $N=3$ to $(N-1)=2$ transition. The goal is to highlight some basic principles by means of a very simple, almost pedestrian, example. The concept can be easily illustrated and explained to a broader audience without advanced 
mathematics using the methods discussed in this short note.

We start by considering the energy levels of a massive particle trapped in a box or lattice in $N=3$ versus $(N-1)=2$ dimensional space. As is well known [9], in 3-d space the energy levels are given by

$$
E^{(3)}=h^{2} / 8 m\left(n_{x}^{2} / L_{x}^{2}+n_{y}^{2} / L_{y}^{2}+n_{z}^{2} / L_{z}^{2}\right)
$$

In Equation (1), $\left(n_{x}, n_{y}, n_{z}\right)$ are quantum numbers, $\left(L_{x}, L_{y}, L_{z}\right)$ are the dimensions of the box in the $(x, y, z)$ direction, respectively, " $h$ " is Planck's constant and " $m$ " refers to the mass of the particle trapped in the box. In 2-d, this reduces to

$$
E^{(2)}=h^{2} / 8 m\left(n_{x}^{2} / L_{x}^{2}+n_{y}^{2} / L_{y}^{2}\right)
$$

In order to keep the discussion simple, we consider a cubic 3-d box where $L_{x}=L_{y}=L_{z}=L$, and correspondingly, $L_{x}=L_{y}=L$ in 2-d space. The superscript on a variable such as the energy, $E$, refers to the space over which the physical quantity is defined.

The lowest energy level in 3-space is $\left(n_{x}, n_{y}, n_{z}\right)=(1,1,1)$ and thus, $E_{111}^{(3)}=3 h^{2} / 8 m L^{2}$; for the same particle in 2-d space, we have $\left(n_{x}, n_{y}\right)=(1,1)$ and therefore $E_{11}^{(2)}=2 h^{2} / 8 m L^{2}$. The next energy level in three dimensional space has a three-fold degeneracy as $\left(n_{x}, n_{y}, n_{z}\right)$ can take on the values, $(1,1,2),(1,2,1)$ or $(2,1,1)$ and this leads to the same energy, $E_{112}^{(3)}=E_{121}^{(3)}=E_{211}^{(3)}=6 h^{2} / 8 m L^{2}$. In two dimensional space, $\left(n_{x}, n_{y}\right)$ can take on the values $(1,2)$ or $(2,1)$; this leads to $E_{12}^{(2)}=E_{21}^{(2)}=5 h^{2} / 8 m L^{2}$, a two-fold degeneracy. We continue in this vein and present our results in table form, Table 1 . In this table, $E_{0}$, is defined by the equation $E_{0}=h^{2} / 8 m L^{2}$, and we consider energies up to, and including $27 / 8 E_{0}$, an arbitrary but sufficient cut-off in energy for our purpose. The degeneracy for a particular energy level is abbreviated as "deg".

As stated, our cut-off in energy was artificially set at $27 E_{0} / 8$; higher energy states are not considered. Specific wave functions can be specified for each of these eigenstates and, in general, the higher the energy level, the more complicated (involved) is the wave function. We now highlight some simple findings. For $N=3$, the total number of energy levels is 27; for $N=2$ spatial dimensions, the corresponding number is only 15 . Furthermore, for $N=3$, we have a greater degeneracy in energy levels, whereas for $N=2$ there is a lessor level of degeneracy, both in terms of number and magnitude. The highest degeneracy for $N=3$ is 6 -fold while for $N=2$, that corresponding number is 3 -fold. The maximum degeneracy for spatial dimension $N$ is given by the equation, max.deg. $(N)=N$ ! .

The entropy will be considered next. According to Boltzmann, the entropy is given by the expression, $S=k_{B} \ln \Omega$, where $k_{B}$ is Boltzmann's constant and $\Omega$ refers to the number of microstate permutations for which an ensemble can be organized to give the same measurable macroscopic result. Consider, for example, $E=17 E_{0} / 8$. According to Table 1 , for $N=3$, we have a three-fold degeneracy for this energy and thus, $S=k_{B} \ln (3)=1.099 k_{B}$. In contrast, for $N=2$, 
Table $1 . N=3$ versus $N=2$ quantized energy states.

\begin{tabular}{|c|c|}
\hline$N=3 \quad$ Spatial Dimensions & $(N-1)=2$ Spatial Dimensions \\
\hline$\left(n_{x}, n_{y}, n_{z}\right)=(1,1,1)$ & $\left(n_{x}, n_{y}\right)=(1,1)$ \\
\hline$\Rightarrow E_{111}^{(3)}=3 h^{2} / 8 m L^{2}=3 E_{0} / 8$ & $\Rightarrow E_{11}^{(2)}=2 E_{0} / 8$ \\
\hline$\left(n_{x}, n_{y}, n_{z}\right)=(1,1,2)=(1,2,1)=(2,1,1)$ & $\left(n_{x}, n_{y}\right)=(1,2)=(2,1)$ \\
\hline$\Rightarrow E_{112}^{(3)}=E_{121}^{(3)}=E_{211}^{(3)}=6 E_{0} / 8$ & $\Rightarrow E_{12}^{(2)}=E_{21}^{(2)}=5 E_{0} / 8$ \\
\hline$(\operatorname{deg} \cdot=3)$ & $(\operatorname{deg} .=2)$ \\
\hline$\left(n_{x}, n_{y}, n_{z}\right)=(1,2,2)=(2,1,2)=(2,2,1)$ & $\left(n_{x}, n_{y}\right)=(2,2)$ \\
\hline$\Rightarrow E_{122}^{(3)}=E_{212}^{(3)}=E_{221}^{(3)}=9 E_{0} / 8$ & $\Rightarrow E_{22}^{(2)}=8 E_{0} / 8$ \\
\hline$($ deg. $=3)$ & \\
\hline$\left(n_{x}, n_{y}, n_{z}\right)=(2,2,2)$ & $\left(n_{x}, n_{y}\right)=(1,3)=(3,1)$ \\
\hline$\Rightarrow E_{222}^{(3)}=12 E_{0} / 8$ & $\Rightarrow E_{13}^{(2)}=E_{31}^{(2)}=10 E_{0} / 8$ \\
\hline$(\operatorname{deg} .=2)$ & $(\operatorname{deg} .=2)$ \\
\hline$\left(n_{x}, n_{y}, n_{z}\right)=(1,1,3)=(1,3,1)=(3,1,1)$ & $\left(n_{x}, n_{y}\right)=(2,3)=(3,2)$ \\
\hline$\Rightarrow E_{113}^{(3)}=E_{131}^{(3)}=E_{311}^{(3)}=11 E_{0} / 8$ & $\Rightarrow E_{23}^{(2)}=E_{32}^{(2)}=13 E_{0} / 8$ \\
\hline$(\operatorname{deg} .=3)$ & $($ deg. $=2)$ \\
\hline$\left(n_{x}, n_{y}, n_{z}\right)=(1,2,3)=(1,3,2)=(2,1,3)=(2,3,1)=(3,1,2)=(3,2,1)$ & $\left(n_{x}, n_{y}\right)=(3,3)$ \\
\hline$\Rightarrow E_{123}^{(3)}=E_{132}^{(3)}=E_{213}^{(3)}=E_{231}^{(3)}=E_{312}^{(3)}=E_{321}^{(3)}=14 E_{0} / 8$ & $\Rightarrow E_{33}^{(2)}=18 E_{0} / 8$ \\
\hline$($ deg. $=6)$ & \\
\hline$\left(n_{x}, n_{y}, n_{z}\right)=(2,2,3)=(2,3,2)=(3,2,2)$ & $\left(n_{x}, n_{y}\right)=(1,4)=(4,1)$ \\
\hline$\Rightarrow E_{223}^{(3)}=E_{232}^{(3)}=E_{322}^{(3)}=17 E_{0} / 8$ & $\Rightarrow E_{14}^{(2)}=E_{41}^{(2)}=17 E_{0} / 8$ \\
\hline$(\operatorname{deg} \cdot=3)$ & $($ deg. $=2)$ \\
\hline$\left(n_{x}, n_{y}, n_{z}\right)=(1,3,3)=(3,1,3)=(3,3,1)$ & $\left(n_{x}, n_{y}\right)=(2,4)=(4,2)$ \\
\hline$\Rightarrow E_{133}^{(3)}=E_{313}^{(3)}=E_{331}^{(3)}=19 E_{0} / 8$ & $\Rightarrow E_{24}^{(2)}=E_{42}^{(2)}=20 E_{0} / 8$ \\
\hline$($ deg. $=3)$ & $(\operatorname{deg} .=2)$ \\
\hline$\left(n_{x}, n_{y}, n_{z}\right)=(2,3,3)=(3,2,3)=(3,3,2)$ & $\left(n_{x}, n_{y}\right)=(3,4)=(4,3)$ \\
\hline$\Rightarrow E_{233}^{(3)}=E_{323}^{(3)}=E_{332}^{(3)}=22 E_{0} / 8$ & $\Rightarrow E_{34}^{(2)}=E_{43}^{(2)}=25 E_{0} / 8$ \\
\hline$(\operatorname{deg} .=3)$ & $(\operatorname{deg} .=2)$ \\
\hline$\left(n_{x}, n_{y}, n_{z}\right)=(3,3,3)$ & $\left(n_{x}, n_{y}\right)=(4,4)$ \\
\hline$\Rightarrow E_{333}^{(3)}=27 E_{0} / 8$ & $\Rightarrow E_{44}^{(2)}=32 E_{0} / 8$ \\
\hline
\end{tabular}

the entropy becomes $S=k_{B} \ln (2)=0.693 k_{B}$. We have less entropy because there is only a 2 -fold degeneracy for that same energy level.

Further insights can be deduced from Table 1. We present these in paragraph/ bullet form.

1) From our simple example, we first notice that space is equivalent to energy 
and vice versa. This is so because we clearly see that a higher dimensional (larger $N)$ space can accommodate, i.e. hold more energy. It simply has more degeneracy, and a higher spectrum of energy levels, than a lower dimensional space for a given cut-off in energy. If we have a finite amount of energy at our disposal, such as $27 E_{0} / 8$, we see that we have 27 energy levels for $N=3$, versus only 15 energy levels for $(N-1)=2$. Furthermore the total energy accommodated in $N=3$ space can be summed up, up to and including, $27 E_{0} / 8$. The result is

$$
\begin{aligned}
E_{\text {TOTAL }}^{(3)}= & {[1 \times 3+3 \times 6+3 \times 9+1 \times 12+3 \times 11+6 \times 14} \\
& +3 \times 17+3 \times 19+3 \times 22+1 \times 27] / 8 E_{0} \\
= & 378 E_{0} / 8
\end{aligned}
$$

where $E_{0}$ was defined as $E_{0}=h^{2} / m L^{2}$. For $(N-1)=2$, the corresponding total energy accommodated, for that same cut-off in energy, is

$$
\begin{aligned}
E_{\text {TOTAL }}^{(2)}= & {[1 \times 2+2 \times 5+1 \times 8+2 \times 10+2 \times 13+1 \times 18} \\
& +2 \times 17+2 \times 20+2 \times 25] / 8 E_{0} \\
= & 208 E_{0} / 8
\end{aligned}
$$

The difference in energy between the two spaces is $E_{\text {TOTAL }}^{(3)}-E_{\text {TOTAL }}^{(2)}=170 E_{0} / 8$, if each space were populated up to the same cut-off energy, $27 E_{0} / 8$.

2) This equivalency between space and energy is a quantum mechanical effect due to the presence of " $h$ " in Equation (1) and Equation (2). In the classical limit where $h \rightarrow 0$, there is no connection between energy given by the left hand side of Equation (1) and Equation (2), and the spatial dimensions of the box, which is specified by the right hand side. The same holds for photons (radiation) as shown in reference [7]. Because the particles are trapped in a box, we have quantized energy levels. As is well known, all bound states have a discrete, versus a continuous, spectrum of energy levels.

3) The higher the spatial dimension, the higher the associated entropy, all other things being equal. $S$ cannot be defined for $N=0$ using the formula, $S=k_{B} \ln \Omega$, because $\Omega=0$. Also, $S=0$ for $N=1$ because there is no degeneracy possible in $1-\mathrm{d}$ space $^{1}[10]$. Here, $\Omega=1$. We count up the total entropy in $N=3$ space, versus $(N-1)=2$ space, for the same cut-off in energy, $27 E_{0} / 8$. For $N=3$, we obtain

$$
S_{\text {TOTAL }}^{(3)}=k_{B}[\ln (6)+6 * \ln (3)]=8.383 k_{B}
$$

${ }^{1}$ We have a similar state of affairs for radiation. The energy density for radiation in $N$ dimensional space is given by the expression:

$u=u(N, T)=2(n-1) \pi^{N / 2}\left(k_{B} T\right)^{N+1} \zeta(N+1) \Gamma(N+1) /\left[(h c)^{N} \Gamma(N / 2)\right]$.

In this equation, $T$ is the temperature in Kelvin, $k_{B}$ is Boltzmann's constant, $c$ equals the speed of light, $h$ is Planck's constant, $\zeta(x)$ is the zeta function, and $\Gamma(x)$ is the gamma function. See references [12] [13] [14] [15]. The entropy density can be expressed in term of the energy density, $u=u(N, T)$; in $N$-dimensional space, we have $s=(N+1) / N u / T$. From these two formulae we notice that for $N=0$, there is no entropy as we are then dividing by $\Gamma(0)$, which is in the denominator and is zero. If $N=1$ is substituted in the above equation, then the denominator is well defined, but we obtain a zero value in the numerator. Radiation energy cannot exist in a 1-dimensional space, and, as a consequence, the entropy likewise equals zero for one spatial dimension. This mirrors what was said for a massive particle. 
To ascertain Equation (5) we have referred to Table 1 . For $(N-1)=2$, the total entropy amounts to

$$
S_{\text {TOTAL }}^{(2)}=k_{B}[6 * \ln (2)]=4.159 k_{B}
$$

This is for the same selected cut-off in energy. We have made use of Table 1 and the relation, $S=k_{B} \ln \Omega$. It is clearly seen that the difference in entropy between the two spaces is $S_{\text {TOTAL }}^{(3)}-S_{\text {TOTAL }}^{(2)}=4.224 k_{B}$, where we assume that each space is populated to the same cut-off energy, namely, $E=27 E_{0} / 8$.

4) For a massive particle, following the steps in reference [7], but now for a massive particle, we claim that

$$
E_{\text {TOTAL }}^{(3)}+S_{\text {TOTAL }}^{(3)} T=E_{\text {TOTAL }}^{(2)}+S_{\text {TOTAL }}^{(2)} T+\Delta Q^{(2)}
$$

Here, $T$ is the temperature in Kelvin, and $\Delta Q$ is any latent heat given off in 2-space as a result of the transition from $N=3$ to $(N-1)=2$. At this stage, $\Delta Q$ can be positive, zero or negative. This is our extension of the CC relation for massive particles in a box. Unlike radiation, there is no pressure component. However, utilizing our above example with the specified cut-off in energy, we obtain from Equation (3), the following result.

$$
378 E_{0} / 8+8.383 k_{B} T=208 E_{0} / 8+4.159 k_{B} T+\Delta Q^{(2)}
$$

Therefore,

$$
\Delta Q^{(2)}=170 E_{0} / 8+4.224 k_{B} T
$$

It is to be noticed that the right hand side is definitely greater than zero for any temperature, $T$, and ground state energy, $E_{0}$. Equation (8) assumes that both spaces are populated to the same specific cut-off energy. Therefore, latent heat must be released in the 2-dimensional space when transitioning from $N=3$ to $(N-1)=2$. If we were to increase the spatial dimension from $(N-1)=2$ to $N=3$, then latent heat would have to be supplied in this amount in order to make the reverse transition. Equation (8) also tells us that the amount of latent heat given off depends specifically on temperature, cut-off energy, and ground state energy.

A simple example might involve electrons trapped in a box of dimensions, $\left(10^{-10}\right.$ $\mathrm{m})$ by $\left(10^{-10} \mathrm{~m}\right)$ by $\left(10^{-10} \mathrm{~m}\right)$, and having total energy $378 E_{0} / 8=1.024 \times 10^{-15} \mathrm{~J}$. All energy levels are filled, and thus 27 electrons are accommodated. We ignore spin and Pauli statistics in order to keep the discussion simple. Upon transitioning to a 2 - $\mathrm{d}$ box of dimensions $\left(10^{-10} \mathrm{~m}\right)$ by $\left(10^{-10} \mathrm{~m}\right), 12$ electrons are expelled, i.e. left in the originating $N=3$ space, due to the fact that only 15 energy states are available in this reduced $(N-1)=2$ space for the specified cutoff energy. Assuming a transition temperature of $293 \mathrm{~K}$ (room temperature), the heat released would be given by Equation (7). We obtain before the spatial transition from 3 -d to 2 -d,

$$
E_{\text {TOTAL }}^{(3)}=378 E_{0} / 8, S_{\text {TOTAL }}^{(3)}=8.383 k_{B}, E_{\text {TOTAL }}^{(2)}=0, S_{\text {TOTAL }}^{(2)}=0
$$

After the spatial transition from 3-d to 2-d, due to the 12 electrons left behind 
in 3-d space, we find that

$$
\begin{aligned}
& E_{\text {TOTAL }}^{(3)}{ }^{\prime}=107 E_{0} / 8, S_{\text {TOTAL }}^{(3)}{ }^{\prime}=3.296 k_{B}, \\
& E_{\text {TOTAL }}^{(2)}{ }^{\prime}=208 E_{0} / 8, S_{\text {TOTAL }}^{(2)}{ }^{\prime}=4.159 k_{B}
\end{aligned}
$$

Primed variables refer to the situation after. The difference gives us $\Delta Q^{(2)}$. By Equation (7), Equation (9) and Equation (10), we see that

$$
\begin{aligned}
\Delta Q^{(2)} & =(378-107-208) E_{0} / 8+(8.383-3.296-4.159) k_{B} T \\
& =1.707 \times 10^{-16}+3.752 \times 10^{-21} \\
& =1.707 \times 10^{-16} \text { Joules }=1.067 \mathrm{keV}
\end{aligned}
$$

This is the release in energy, in 2-d space, for the transition of the 15 electrons from 3-d to 2-d space, and we can definitely see that this is a positive release of energy. The 12 electrons that cannot be accommodated in 2-d space occupy the lowest states in 3-d space. Particles trapped in a two dimensional box or lattice have less degrees of freedom, than corresponding particles trapped in a 3-box or lattice for the same cutoff energy. This manifests itself in the release of latent heat through Equation (7) and Equation (11).

5) Two limits can take us from the $N$-dimensional space to the $(N-1)$ -dimensional space. The first limit involves taking the length parameter, $L_{z}$ in Equation (1), and have this length approach infinity ${ }^{2}$ [11]. The second limit is to let $L_{z}$ approach zero. From an energy standpoint, the $2^{\text {nd }}$ limit makes more sense.

We consider the transition $N=3$ to $(N-1)=2$, where Equation (1) reduces to Equation (2). In both limits, the " $Z$ " space is weighed differently, versus $X$ and $y$ coordinates. In the first limit, the " $z$ " space is stretched out, whereas in the second limit, the " $Z$ " space is shortened, i.e. compactified. If the box in the " $Z$ " direction is stretched out, then there can be little quantization in the $z$ sense, as we will have close to zero energy levels in this direction in space. In one dimension, the energy is quantized, $E_{n}=p_{n}^{2} / 2 m$ where $p_{n}$ is the momentum of the particle. Because of the de Broglie relation, we know that $p_{n}=h / \lambda_{n}$, where $\lambda_{n}$ is the wavelength. When confined to a box of width $L$, this gives for the quantized energy levels, $E_{n}=n^{2} h^{2} /\left(8 m L^{2}\right)$. From this expression it is seen that in the limit where $L$ approaches infinity, $E_{n}$ must approach zero. Moreover, as the temperature decreases, we might expect a spatial transition. If this occurs, then the $z$-space has lower energy levels in this limit, where $L_{z} \rightarrow \infty$. However, even though the quantized energy levels in $z$-space go down relative to the $x$ - $y$-space, the expanse of $z$-space goes up. In fact, it approaches infinity. We therefore expect an overall increase in total energy when 3 - $d$ space transitions to 2 - $d$ space when taking this limit. But based on our arguments above, and the entries given in Table 1, this is clearly not so. Energy has been given off as latent heat upon transitioning from a higher dimension to a lower one, and this is only possible if we had more energy to begin with than after. Thus the $x-y$-z-space must have more energy versus the $x$ - $y$ space $+z$-space, separately, after the transition.

${ }^{2}$ This would correspond to the de Sitter radius $R \rightarrow \infty$ and the rotation group, $S O(1,4) \rightarrow S O(1,3)$. 
A better limit to take is to let $L_{z}$ approach zero. The $z$-space is now confined to a narrower width, and, as a consequence, there is more quantization in the $z$-sense, i.e. the energy levels get bumped up to where they, perhaps, are no longer readily observable. However, the physical realm length-wise, over which they act, is much less. The $x-y$ space is now occupied by lower energy levels than the $z$-space energy levels, since the $z$ space width is much narrower. When plotting quantized energy levels as a function of dimension, each dimension of space has a specific width or weight. This is the realm over which the quantized energy levels can be found. Associated with a spatial dimension is quantized energy, and each has a corresponding height. If the width associated with one particular spatial direction freezes upon transitioning, then the associated energy levels must stay fixed. Therefore, the energy levels in that particular spatial direction, the compactified direction, can no longer decrease.

Another way of arguing the same thing is as follows. Before the transition, all $x-y-z$ components are weighed equally. And all have the same fixed energy levels. As the temperature decreases, the quantized energy levels decrease in $x-y-z$ directions until, at some critical temperature, a transition from 3 -d space to $2-\mathrm{d}$ space occurs. The $x$ - $y$ space continues to decrease in terms of weight, but the $z$-space width has frozen since positive energy is released in the form of latent heat. We saw that to be the case by our model example. If, on the other hand, the $z$-space weight were to increase and the width gets larger, then there would be more energy after, versus before, within the space itself. This is not compatible for a positive release of latent heat as demonstrated above.

A specific example might be the early universe, where a hypothetical $N=4$ to $(N-1)=3$ transition may have occurred [8]. Assuming such a model, as the universe cools upon expansion, the energy levels must decrease due to box quantization. Early on, each of the 4-dimensions must have been occupied with rather higher energy levels, having been confined to a relatively small volume. At some critical temperature, which we denote by $T_{43}$, a transition occurs from 4 -space to 3 -space. Latent heat is given off and the weight of the $4^{\text {th }}$ dimension, the $\mathrm{w}$-space, has compactified, i.e. frozen its width. The quantized energy levels continue to decrease for the remaining $x-y-z$ spatial dimensions, and we only observe 3-d expansion upon decoupling. An estimate of the compactification length was given in a previous work [7]. The reason we may not see the energy levels associated with the $4^{\text {th }}$ dimension in this transition, and the reason we do not appreciate the lowering of such energy levels due to expansion, is because our modern day accelerators have not yet reached, and perhaps will never reach, such small compactification scales.

6) The $(N-1)$ volume is a subset of the $N$ volume when compactification occurs. Again, consider our particle in the box example, and again let us focus on the $N=3$ to $(N-1)=2$ transition. It is obvious that $V^{(3)}=L_{x} L_{y} L_{z}=V^{(2)} L_{z}$. In the first limit, $V^{(3)}$ approaches infinity in cubic meters, whereas, in the second limit, $V^{(3)}$ approaches zero, also in cubic meters. $V^{(2)}$, on the other hand, is measured in square meters, and being a different dimensional quantity, $V^{(2)}$ 
cannot be compared to $V^{(3)}$ directly. The ratio, $V^{(3)} / V^{(2)}$, is a measure for the weight of the space allocated to $L_{z}$.

In a previous paper [7], we argued that the universe may have once had 4 spatial dimensions in a very early epoch and the 3-d world we see now may be a special case limit. The $4^{\text {th }}$ dimension has curled up on itself a long time ago, and will only be visible once higher accelerator energies are built. Within the greater universe itself at present, such pockets of 4-d space may still exist, and be only visible from the outside, such as in a black hole. The temperature plays a key role in any first order phase transition, and here it would be no different. If, for example, $V^{(4)}$ equals $10^{-80} \mathrm{~m}^{4}$, but $V^{(3)}$ equals $10^{-54} \mathrm{~m}^{3}$, then the compactified length, $L_{w} \equiv V^{(4)} / V^{(3)}=10^{-26} \mathrm{~m}$. This would be an unobservable world for which we have little direct knowledge or appreciation.

7) Energy must be conserved when transitioning between different dimensions of space. We next consider a transition from $N=4$ to $(N-1)=3$, and rewrite our generalized CC equation, Equation (7), as

$$
E_{\text {TOTAL }}^{(4)}+S_{\text {TOTAL }}^{(4)} T=E_{\text {TOTAL }}^{(3)}+S_{\text {TOTAL }}^{(3)} T+\Delta Q^{(3)}
$$

where $\Delta Q^{(3)}$ is the latent heat released in 3-d space. We know that $\Delta Q^{(3)}$ must be positive. Dividing Equation (12) by the 3-Volume, $V^{(3)}$, the equation takes the form

$$
\left(u_{\text {TOTAL }}^{(4)}+s_{\text {TOTAL }}^{(4)} T\right) V^{(4)} / V^{(3)}=u_{\text {TOTAL }}^{(3)}+s_{\text {TOTAL }}^{(3)} T+\Delta q^{(3)}
$$

In Equation (13), $V^{(4)}$ is the 4-Volume, $u^{(4)}$ and $u^{(3)}$ are the internal energy densities in 4-d and 3-d space, respectively, and $s^{(4)}$ and $s^{(3)}$ are the entropy densities in 4-d and 3-d space, respectively. Finally we have the latent

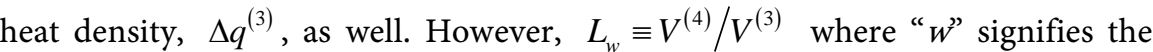
$4^{\text {th }}$ dimension. Hence, Equation (13) assumes the form

$$
\left(u_{\text {TOTAL }}^{(4)}+s_{\text {TOTAL }}^{(4)} T\right) L_{w}=u_{\text {TOTAL }}^{(3)}+s_{\text {TOTAL }}^{(3)} T+\Delta q^{(3)}
$$

This equation is dimensionally consistent even though, in terms of units, $\operatorname{dim}\left[u^{(4)}\right]=\mathrm{J} / \mathrm{m}^{4}$, which is unequal to $\operatorname{dim}\left[u^{(3)}\right]=\mathrm{J} / \mathrm{m}^{3}$. Moreover, $\operatorname{dim}\left[s^{(4)}\right]=\mathrm{J} /\left(\mathrm{m}^{4} \cdot \mathrm{K}\right)$ is unequal to $\operatorname{dim}\left[\mathrm{s}^{(3)}\right]=\mathrm{J} /\left(\mathrm{K} \cdot \mathrm{m}^{3}\right)$. Equation (14) allows us to solve for $L_{w}$ if we can determine the other quantities. $L_{w}$ is obviously dependent on $\Delta q^{(3)}$, as can be seen by Equation (14).

In all likelihood, Equation (14) will not be that useful for massive particles as it is difficult to imagine how one can determine energy densities for massive particles in 4-d space. However, for massless particles, i.e. radiation, it is extremely useful. For radiation the internal energy densities, the pressure, and the entropy densities are readily determined as functions of spatial dimension, and temperature [8] [12] [13] [14] [15]. We have to include pressure contributions on both left and right hand sides for black-body photons, but these are also readily known. In fact, entropy density and pressure are multiples of internal energy density where the numerical factor depends strictly on the spatial dimension considered. As a consequence, the compactification parameter $L_{w}$ is thus 
strictly determined by the amount of latent heat given off as shown in a previous work [7], where we used a modified, i.e. extended version of Equation (14), which applies for radiation. The $L_{w}$ versus $\Delta q^{(3)}$ dependency for radiation is a linear relationship. This is also true for Equation (14), which holds for massive particles.

In conclusion, by means of a simple, almost elementary example, we have shown that space has energy content. In transitioning from a higher dimensional space to a lower one, latent heat is invariably released and a generalized CC relation holds. We focused on massive particles in a box, in $N$ dimensions, and then again, in $(N-1)$ dimensions. The identity of the particle, given by its mass, remained constant and the only thing that changed was the dimension of the box itself. A higher dimensional space can accommodate more particles, and at a higher energy, than a lower dimensional space. In transitioning between spatial dimensions, energy therefore must be released. Moreover, two limits are possible, but only the compactified limit, $L_{w} \rightarrow 0$, seems to make sense from a conservation of energy viewpoint. We saw that as a spatial dimension freezes or compactifies, quantized energy levels in the compactified space dimension stay large and, mostly likely, beyond view. However, the weight or width of remaining non-compactified space increases even though a dimension of space has curled up.

\section{Acknowledgements}

The author wishes to thank John Krehbiel for numerous and interesting discussions on this point.

\section{Conflicts of Interest}

The author declares no conflicts of interest regarding the publication of this paper.

\section{References}

[1] Klein, O. (1926) Quantentheorie und Fünfdimensionale Relativitätstheorie. Zeitschrift für Physik, 37, 895-906.

[2] Jordan, P. (1948) Fünfdimensionale Kosmologie. Astronomische Nachrichten, 276, 193-208.

[3] Appelquist, T., Chodos, A. and Freund, P.G.O. (1987) Modern Kaluza-Klein Theories. Addison-Wesley, Menlo Park.

[4] Wesson, P.S. (1999) Space-Time-Matter, Modern Kaluza-Klein Theory. World Scientific, Singapore. https://doi.org/10.1142/3889

[5] Wesson, P.S. and Ponce de Leon, J. (1995) The Equation of Motion in Kaluza-Klein Cosmology and Its Implications for Astrophysics. Astronomy and Astrophysics, 294, 1-7.

[6] Castellani, L., et al. (1991) Supergravity and Superstrings. Vol. 2, Chapter V.11, World Scientific, Singapore.

[7] Pilot, C. (2019) A New Type of Phase Transition Based on the Clausius-Clapeyron 
Relation Involving a Change in Spatial Dimension. Journal of High Energy Physics, Gravitation and Cosmology (JHEPGC), Vol. 5.

[8] Gonzalez-Ayala, J., Cordero, R. and Angulo-Brown, F. (2015) Is the $(3+1)-d \mathrm{Na}-$ ture of the Universe a Thermodynamic Necessity? EPL (Europhysics Letters), arXiv:1502.01843v2.

[9] Serway, R., Moses, C. and Moyer, C. (2005) Modern Physics. 3rd Edition, Thomson Learning, Inc., Toronto, 260-266.

[10] Greiner, W., Neise, L. and Stöcker, H. (1995) Thermodynamics and Statistical Mechanics. Springer, Berlin, 130-131

[11] Dooley, A.H. and Rice, J.W. (1985) On Contractions of Semisimple Lie Groups. Transactions of the American Mathematical Society, 289, 185-202.

https://doi.org/10.1090/S0002-9947-1985-0779059-4

[12] Landsberg, P.T. and De Vos, A. (1989) The Stefan Boltzmann Constant in an n-Dimensional Space. Journal of Physics A: Mathematics and General, 22, 1073-1084. https://doi.org/10.1088/0305-4470/22/8/021

[13] Menon, V.J. and Agrawal, D.C. (1998) Comment on "the Stefan-Boltzmann constant in n-Dimensional Space”. Journal of Physics A: Mathematical and General, 31, 1109-1110. https://doi.org/10.1088/0305-4470/31/3/021

[14] Barrow, J.D. and Hawthorne, W.S. (1990) Equilibrium Matter Fields in the Early Universe. Monthly Notices of the Royal Astronomical Society, 243, 608-609.

[15] Gonzalez-Ayala, J., Perez-Oregon, J., Cordero, R. and Angulo-Brown, F. (2015) A Possible Cosmological Application of Some Thermodynamic Properties of the Black Body Radiation in n-Dimensional Euclidean Spaces. Entropy, 17, 4563-4581. https://doi.org/10.3390/e17074563 